\title{
A Simple Sparse Channel Estimation Method for Single-Carrier Frequency Domain Equalization Based on Noise Space
}

\author{
Yuanhong Zhong, Yao Zhou and Qilun Lei \\ College of Communication Engineering, Chongqing University \\ Chongqing, 400044, China \\ zhongyh@cqu.edu.cn
}

\begin{abstract}
It is widely accepted that SC-FDE (Single-Carrier Frequency Domain Equalization) is an excellent candidate for broadband wireless systems. Channel estimation is one of the key challenges in SC-FDE, since accurate channel estimation can significantly improve the equalization at the receiver and consequently enhance the communication performances. In this paper, we proposed a simple sparse channel estimation method for $S C-F D E$ system based on noise space, through sorting the results of the Least-squares (LS) channel estimation, channel tap locations and value are evaluated. The proposed system can realize channel estimation at a very low complexity, and simulation result shows that it can achieve significantly improved performance in frequency selective fading sparse channel.
\end{abstract}

Keywords: Noise Space, SC-FDE, Sparse Channel Estimation, Sorting

\section{Introduction}

In a typical wireless scenario, the transmitted signal arrives at the receiver via various paths of different lengths. This leads to inter-symbol interference (ISI) and posts a major difficulty to information decoding. And traditional single carrier modulation with time domain equalization approach is unattractive, due to the high complexity of the receiver and required signal processing time. However, the frequency domain equalization system is a good choice, for example, orthogonal frequency division multiplexing (OFDM) [1]. OFDM has been widely applied in wireless communication systems because it transmits at a high rate, achieves high bandwidth efficiency, and is relatively robust to multipath fading and delay, but it suffers from a large peak to average power ratio (PAPR).

On the other hand, single carrier modulation combined with frequency domain equalization [2], which attempts to approach the performance and complexity of OFDM while maintaining a lower PAPR compared to OFDM, is another excellent technology for broadband systems. Single-Carrier Frequency Domain Equalization (SC-FDE) with cyclic prefix is a single carrier technique that has lately received much attention and is an alternative to orthogonal frequency division multiple access for $4 \mathrm{G}$ technology. And its frequency-division multiple access scheme Single-carrier FDMA (SC-FDMA) has been adopted for uplink transmission technique in both 3GPP Long Term Evolution (LTE) and LTE Advanced standards [3]. The most attractive advantage of SC-FDE is its low PAPR. Since most of the cost in communication terminals comes from the power amplifier, a lower PAPR can significantly reduce the costs of mobile units. Moreover, it makes the mobile units' power more efficient. Since the orthogonal frequency division multiple access (OFDMA) is used in the downlink, both the burdens of complex frequency domain equalizer needed for the SC-FDMA and accommodating large PAPR in OFDMA rest upon the base station.

Channel estimation is critical to the performance of coherent SC-FDE demodulation. It 
also relates to diversity reception, the design of optimum match receiver, and adaptive link technologies, which all require a good channel estimation support. Therefore, accurate channel estimation can significantly improve the SC-FDE system performances [1-3]. Numerous experimental studies undertaken by various researchers in the recent past have shown that wireless channels associated with a number of scattering environments tend to exhibit sparse structures in the sense that majority of the channel taps end up being either zero or below the noise floor when operating at large bandwidth and symbol duration and/or with large plurality of antennas $[4,5]$. However, traditional training-based methods that rely on linear reconstruction schemes at the receiver seem incapable of exploiting the inherent low dimensionality of such sparse channels, thereby leading to over utilization of the key communication resources of energy, latency, and bandwidth. A number of researchers have tried to address this problem in the recent past and proposed training signals and reconstruction strategies that are tailored to the anticipated characteristics of sparse multipath channels $[4,5]$, but these methods require a lot of computing. Recently, there has been a growing interest in considering using compressed sensing theory in pilot-assisted sparse channel estimation to reduce the number of pilot symbols. In [6-9], the CS (Compress Sensing) theory has been employed for sparse channel estimation in OFDM and single-carrier systems. The method based CS theory makes the less use of pilot which means more efficient system resources. However, the recover algorithm of CS often needs large amounts of computing. This paper presents a simple sparse channel estimation method for SC-FDE based on noise space, through sorting the results of the Least-squares (LS) channel estimation, channel tap locations and value are evaluated, and the proposed system can realize channel estimation at a very low complexity.

The rest of paper is organized as follows. In section II we review the general SC-FDE system model and give the problem statement. In section III we will present the simple sparse channel estimation method for SC-FDE system based on noise space in detail. Section IV discusses the results of the computer simulation, and conclusions are given in Section V.

\section{Problem Statement}

\subsection{SC-FDE System}

Figure 1 shows the block diagram of SC-FDE system ${ }^{[2]}$, each group of consecutive information bits is mapped into a complex symbol belonging to a m-ary complex constellation, then with $\mathrm{N}$ symbols producing a data block, each block is cyclically extended, inserting a repetition of its last symbols at its beginning as shown in Figure 2. In some systems alike, the cyclic prefix (CP) can be replaced by unique-word. At the receiver, the $\mathrm{CP}$ of the block is first deleted, and then the frequency domain equalization is used as OFDM system, finally symbols are demapped and outputted.

To reach good performance of frequency domain equalization, the fading channel information must be estimated, and the estimation performance is critical to SC-FDE system, since channel estimation also relates to diversity reception, and adaptive link technologies, and so on. Channel estimation can be divided into two categories ${ }^{[1]}$, blind and non-blind. Based on consideration of the complexity and performance, non-blind channel estimation, i.e. channel estimation with pilot-aids, is usually adopted. The pilot can be inserted in frequency domain or in time domain, and inserting a pilot in frequency domain is used in case of continuous data transmission. For burst data block or the first data block in system, training sequences or training symbols are generally used. A training sequence is a block in SC-FDE whose whole block is used for channel estimation as shown in Figure 2. In this paper, we will discuss the sparse channel estimation method of SC-FDE using the training sequence. 


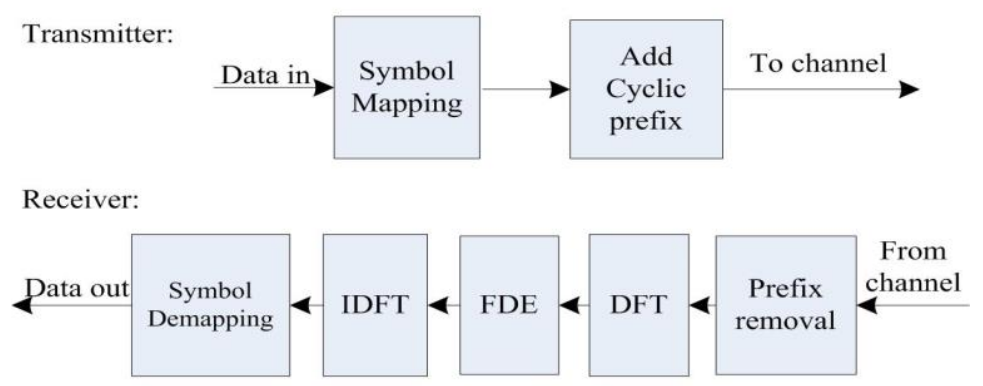

Figure 1. Block Diagram of SC-FDE

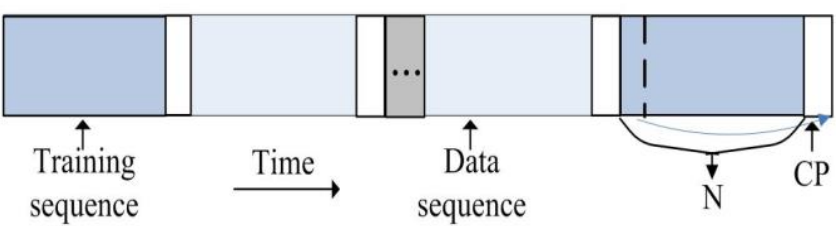

Figure 2. Frame Structure of SC-FDE

\subsection{Sparse Channel Estimation}

Consider a SC-FDE system which has a training symbol block with size $\mathrm{N}$, the training sequence is denoted as $\mathbf{d}=\left[d_{0} d_{1} \ldots d_{N-1}\right]^{T}$, and the block is transmitted after adding $\mathrm{CP}$, with the length of $\mathrm{CP}$ greater than the length of the channel impulse response. At the receiving end, after synchronization and removing the $\mathrm{CP}$, it is convenient to get:

$$
\mathrm{y}=\mathrm{Dh}+\mathrm{w}
$$

Where $\mathrm{y}$ is reception block signal in time-domain sequence after deleting $\mathrm{CP}$, and $\mathbf{y}=\left[\begin{array}{llll}y_{0} & \mathrm{y}_{1} & \ldots \mathrm{y}_{N-1}\end{array}\right]^{T} . \mathbf{W}$ is the Gaussian white noise with mean value zero and variance $\delta_{w}$. $\mathbf{D} \in C^{\mathrm{NXN}}$ is cyclic matrix with $d_{0}$ as the value of the main diagonal and the first row is $\left[d_{0} d_{N-1} d_{N-2} \ldots d_{1}\right] . \mathbf{h}=\left[h_{0} \mathrm{~h}_{1} \ldots \mathrm{h}_{L-1} 0 \ldots 0\right]^{T}$ is the NX1 channel impulse response. Since $\mathrm{D}=\mathrm{F}^{-1} \mathrm{XF} \quad$, where $\mathbf{X}=\operatorname{diag}(F(\mathbf{d})), \quad \mathrm{F}$ is the NXN DFT matrix, $[F]_{n k}=\frac{1}{\sqrt{N}} \exp (-j 2 \pi n k / N), k 、 n=0,1, \ldots \mathrm{N}-1$, and $\mathrm{F}^{-1}$ is the NXN inverse DFT matrix, the reception signals in the frequency domain can be denoted as:

$$
\mathrm{Y}=\mathrm{XH}+\mathrm{W}=\mathrm{XFh}+\mathrm{W}
$$

Where $\mathrm{Y}, \mathrm{W}$ is the reception signals and Gaussian white noise in the frequency domain. According to equation (2), the LS channel estimation can be obtained directly ${ }^{[10]}$ :

$$
\hat{\mathrm{h}}_{\mathrm{LS}}=\left[\hat{\mathrm{h}}_{0}, \ldots, \hat{\mathrm{h}}_{\mathrm{N}-1}\right]^{\mathrm{T}}=\mathrm{F}^{-1} \mathrm{X}^{-1} \mathrm{Y}
$$

In order to further improve performance, the literature ${ }^{[11]}$ proposed a modified LS algorithm (mLS). It takes advantage of the feature that channel length does not exceed the cyclic prefix length, its estimated value is:

$$
\hat{\mathrm{h}}_{\mathrm{mLS}}=\left(\mathrm{F}_{1}^{\mathrm{H}} \mathrm{X}^{\mathrm{H}} \mathrm{XF}_{1}\right)^{-1} \mathrm{~F}_{1}^{\mathrm{H}} \mathrm{X}^{\mathrm{H}} \mathrm{Y}
$$

where $F_{1}$ is a $N X N_{c p}$ DFT matrix, and

$$
\left[F_{1}\right]_{p q}=\frac{1}{\sqrt{N}} \exp (-j 2 \pi p q / N)
$$

$$
p=0,1, \ldots, N-1, q=0,1, \ldots, N_{C P}-1 \text {. }
$$

However, when the channel characteristic is specified as sparsity, their estimation performance cannot be satisfying because LS algorithm does not take advantage of the properties of sparse channel. For sparse channel estimation, literature ${ }^{[12]}$ gives a simple estimation algorithm based on the signal energy testing. The algorithm estimates the 
channel using the LS at first, and supposes the real channel is composed of the estimated value and the estimation error. Therefore $\hat{h}_{L S}(n)=h(n)+e(n), n=0,1, \ldots N-1$, where $e(n)$ is the Gaussian white noise with mean value zero and variance $\delta_{e}$. In order to improve the channel estimation value, the channel length $\hat{L}$ is estimated. Finally the channel taps which are greater than the length of estimate are set to zero. Energy detection algorithm is simple, but the estimation performance is unsatisfactory, therefore literature ${ }^{[13]}$ proposed a GAIC algorithm based on Generalized Akanke Information Criterion (GAIC) in OFDM system. This algorithm uses a manner of iteration. First, the channel length is estimated based on the Cost function of GAIC, and then remove the impact of last channel path and set it to zero, after then the training sequence is again pass through the channel and reestimate the channel length with iteration, finally the position of channel taps can be estimated. With this information the channel estimation can be improved as:

$$
\hat{H}_{i L S}=F_{2}\left(F_{2}^{H} X^{H} X F_{2}\right)^{-1} F_{2}^{H} X^{H} Y
$$

Where $F_{2}$ is composed of the column of DFT which is the nonzero channel tap, that means $\left[F_{2}\right]_{p q}=\frac{1}{\sqrt{N}} \exp (-j 2 \pi p q / N), p=0,1, \ldots, N-1, q \in\left\{0,1, \ldots, N_{C P}-1\right\}$. Then through the inverse Fourier transform, the modified channel impulse response can be obtained.

The performance of GAIC algorithm is excellent, but due to the nature of the algorithm is iterative, it has high complexity. There are many literatures on sparse channel estimation problems, such as sparse channel estimation based on CS theory and so on. These algorithms may have high complexity, or poor performance, and maybe require a priori information about the channel, therefore algorithm performance cannot be satisfied. By resorting to the idea of sorting ${ }^{[14]}$, this paper proposed a sparse channel estimation method by sorting in noise space. This algorithm not only has better performance, but is easy to implement too.

\section{The Proposed Sparse Channel Estimation Method}

The proposed sparse channel estimation method is based on sorting the results of the estimation of LS in noise space. Firstly, LS estimation algorithm is used as an initial estimate of the channel, then sort the initial value $\hat{\mathbf{h}}_{L S}$ in ascending order, which can be denoted as:

$$
\hat{\mathbf{h}}_{L S}^{\prime}=\left[\hat{h}_{i(0)}, \hat{h}_{i(0)}, \ldots, \hat{h}_{i(N-1)}\right]^{T}
$$

And store the index of taps:

$$
I=[i(0), i(0), \ldots, i(N-1)]
$$

The purpose of doing so is based on that if the channel taps are nonzero channel taps, then the amplitude value of nonzero taps should have a greater likelihood of greater value. Therefore, once the numbers of channel taps are determined, the nonzero tap positions of the channel can be determined. In order to find out the number of nonzero taps, a vector variance $\Phi$ is defined as follows:

Where

$$
\Phi=\left[\phi_{0}, \phi_{1}, \ldots, \phi_{\mathrm{K}}, \ldots \phi_{N-1}\right]
$$

$$
\phi_{K}=\frac{1}{K+1} \sum_{k=0}^{K}\left|\hat{h}_{i(k)}\right|^{2}, 0 \leq K \leq N-1
$$

Since $\hat{\mathbf{h}}_{L S}$ is sorted in ascending order, and provided the block length $\mathrm{N}$ is long enough, when the $K$ increases, $\phi_{K}$ also increases, and when $K=N-V-1$, the value of $\phi_{K}$ should be equal to the average variance of the noise $\delta_{w}^{2}$. Following this features, we can discriminate the noise space and the signal space according to[14]: 


$$
\delta_{w}^{2} \leq \phi_{\hat{V}}, \text { and } \phi_{\hat{V}-1} \leq \delta_{w}^{2}
$$

It means if formula (10) is met, the $\hat{V}$ would be the number of nonzero channel taps, and the last $N-\hat{V}$ elements of $\hat{\mathbf{h}}_{L S}^{\prime}$ given by $\{i(\hat{V}), \ldots, i(N-1)\}$ are the nonzero channel taps, other taps can be set to zero. The final estimate can be expressed as:

$$
\hat{H}_{N m L S}=F_{3}\left[F_{3}^{H} X^{H} X F_{3}\right]^{-1} F_{3}^{H} X^{H} Y
$$

Where $\left[F_{3}\right]_{p q}=\frac{1}{\sqrt{N}} \exp (-j 2 \pi p q / N), p=0,1, \ldots, N-1, q=i(\hat{V}), \ldots, i(N-1)$ is a partial Fourier matrix which has only the nonzero taps column .

In the above algorithm, it is necessary to know the channel noise $\delta_{w}^{2}$ in advance, and it can be obtained by calculating the taps which is larger than the cyclic prefix from formula (2). The noise in time domain can be defined as:

$$
\hat{\mathbf{w}}=\left[\hat{w}_{0}, \ldots, \hat{w}_{N-N_{c p}-1}\right]^{T}=F_{4}\left[F_{4}^{H} X^{H} X F_{4}\right]^{-1} F_{4}^{H} X^{H} Y
$$

Where $\left[F_{4}\right]_{p q}=\frac{1}{\sqrt{N}} \exp (-j 2 \pi p q / N), \quad p=0,1, \ldots, N-1, q=N_{c p}, N_{c p+1}, \ldots, N-1$, is a partial Fourier matrix which is composed of the last $N-N_{c p}$ column. The average noise variance can be get:

$$
\delta_{w}^{2}=\frac{1}{N-N_{C P}} \sum_{i=0}^{N-N_{C P}-1}\left|\hat{w}_{i}\right|^{2}
$$

If the last element of $N-\hat{V}$ has an index greater than the cyclic prefix, it can be set to zero directly, since the length of channel response does not exceed the length of the cyclic prefix.

\section{Simulation Results}

In this section, Monte Carlo simulations are conducted to show the channel estimation performance of the proposed algorithm. To begin with, assume that symbols block in the system of SC-FDE is time-invariant, the length of symbol block $N=256$, the maximum channel length $L=64$, training sequence used is the Zadoff-Chu sequence as recommended in the LTE. The multipath channel has a characteristic of negative exponential power delay, that is, each path power is $\delta_{l}^{2}=\exp \left(-4 l / N_{c p}\right), l=0,1, \ldots L-1$, where $N_{c p}=64$ is the length of cyclic prefix. The number of Nonzero channel taps $V=4$. System synchronizes to the first path, which means that $h_{0}$ is always nonzero, and other path delays are randomly distributed over the entire channel length $L$. In order to evaluate the performance of the channel estimation algorithm, we define the normalized mean square error (Normalized mean square error, NMSE) of the channel estimation as follows:

$$
\text { NMSE }=\frac{E\left\{\sum_{k}\left|H_{k}-\hat{H}_{k}\right|^{2}\right\}}{E\left\{\sum_{k}\left|H_{k}\right|^{2}\right\}}
$$

Figure 3 compares the NMSE performance of the proposed algorithm with the LS, $\mathrm{mLS}$ and GAIC versus signal-to-noise ratio (SNR). It is seen that the LS has the worst performance, because it does not use features of sparse channel, and $\mathrm{mLS}$ algorithm does better for the removal of noise in a block. The proposed algorithm has similar performance with GAIC. Moreover, in high SNR, the proposed algorithm has more than about $1 \mathrm{~dB}$ gain against GAIC. Furthermore, the proposed estimation algorithm has relatively low complexity, it just needs sorting, comparing and the Fourier transform 
operation, in contrary, the GAIC requires iteration. Therefore the proposed algorithm based on sorting in noise space is more suitable for practical application.

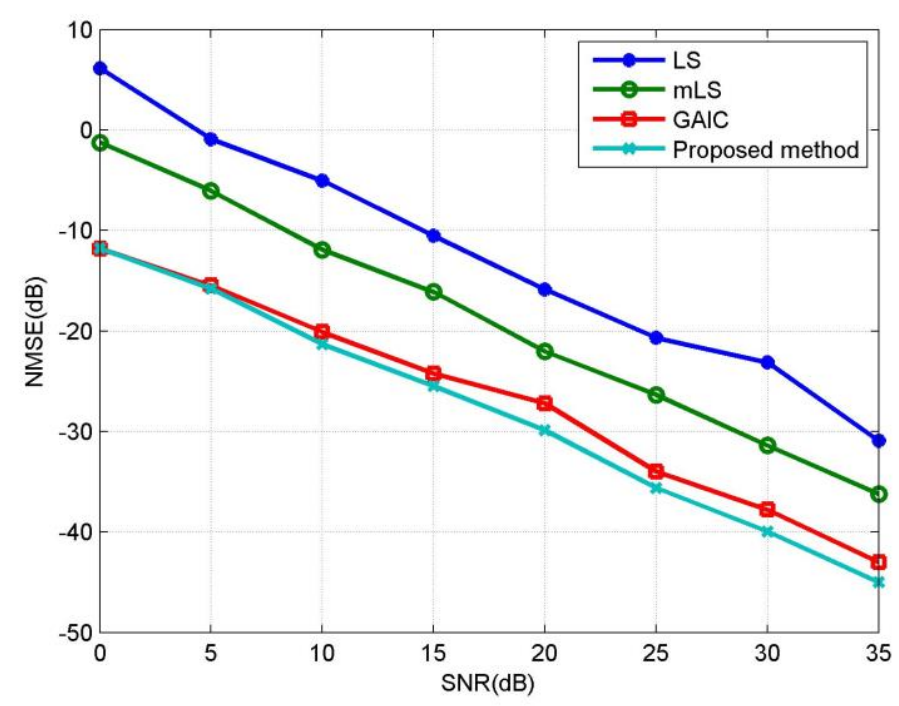

Figure 3. Estimating Performance Comparison of Various Algorithms

Figure 4 depicts the Symbol error rate (SER) performance of the proposed approach and other methods in SC-FDE system. In the simulation, single-point zero forcing equalizer is adopted. As can be seen from Figure 4, the performance of the proposed algorithm is very close to ideal channel which indicates that the algorithm is effective.

The training data after deleting $\mathrm{CP}$ of a block is used to estimate noise variance in the algorithm. When the block length is small, the estimated variance does not necessarily reflect the real channel noise variance. To evaluate the impact of the block length on the performance of the proposed approach, the same experiment as described above is repeated for different block length. Figure 5 shows the performance of the system under different block length. Note that increasing the number of training data, i.e. increasing the block length, improvement of the channel noise variance estimation is achieved, which leads to a performance gain. Therefore, there is a trade-off between performance and efficiency, appropriate length needs to be selected.

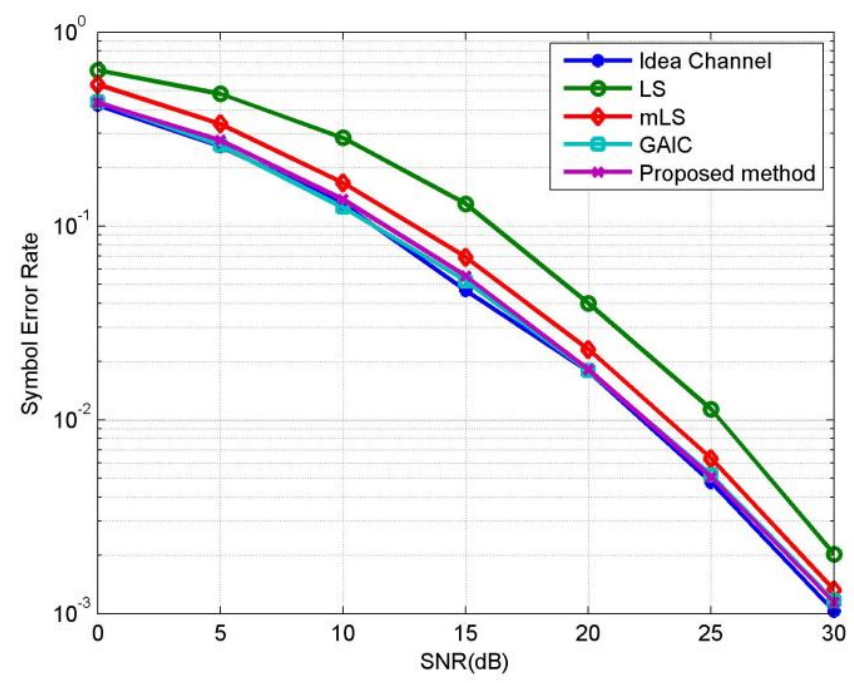

Figure 4. Comparison of SER using different Channel Estimation Methods 


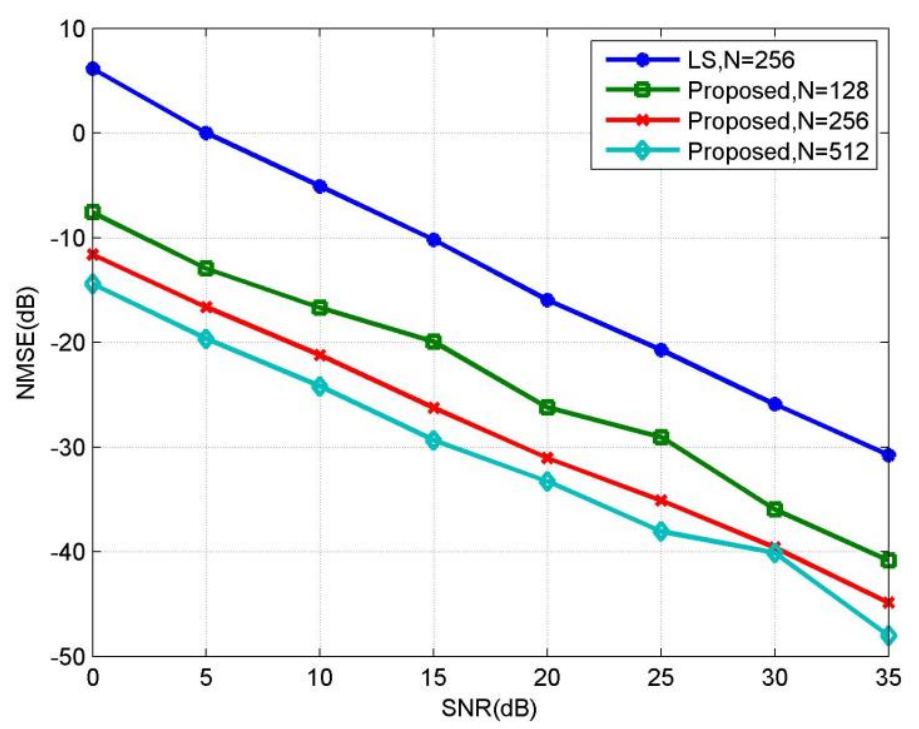

Figure 5. Influence of the Estimation Performance under Different Block
Length

\section{Conclusion}

In this paper, a simple sparse channel estimation method for SC-FDE system based on noise space is proposed, through sorting the results of the Least-squares channel estimation, channel tap locations and there value are estimated. The proposed system can realize channel estimation at a very low complexity, and simulation result shows that it can achieve significantly improved performance in frequency selective fading sparse channel and it is well suited for applications in wireless SC-FDE system.

\section{Acknowledgment}

This work is supported by the Fundamental Research Funds for the Central Universities under Grant No. CDJZR12160015 and No. CQDXWL-2013-010. The paper is also supported by Key Program of Natural Science Foundation of Chongqing under Grant No. CSTC2013JJB40004 and National Natural Science Foundation of China under Grants No. 11205254.

\section{References}

[1] J. G. Proakis and M.Salehi, "Digital Communication", McGraw-Hill, 5th Edition, (2007).

[2] D. Falconer, S. L. Ariyavisitakul and A. Benyamin-Seeyar, "Frequency Domain Equalization for SingleCarrier Broadband Wireless Systems”, IEEE Communication Magazine, vol. 40, no. 4, (2002), pp. 58 66.

[3] 3GPP TS 36.201 V 11.1.0, LTE-Physical Layer-General description (Release 11), (2013).

[4] S. F. Cotter, B. D. Rao, "Sparse channel estimation via matching pursuit with application to equalization, IEEE Transactions on Communications, vol. 50, no. 3, (2002), pp. $374-377$.

[5] C. Carbonelli, S. Vedantam and U. Mitra, "Sparse Channel Estimation with Zero Tap Detection, Wireless Communications", IEEE Transactions on Wireless Communications, vol. 6, no. 5, (2007), pp. $1743-1763$.

[6] W. U. Bajwa, J. Haupt, A. M. Sayeed and R. Nowak, "Compressed Channel Sensing: A New Approach to Estimating Sparse Multipath Channels", Proceedings of the IEEE, vol. 98, no. 6, (2010), pp. 1058 1076.

[7] C. R. Berger, Z. H. Wang and J. Z Huang, "Application of compressive sensing to sparse channel estimation”, IEEE Communications Magazine, vol. 48, no. 11, (2010), pp .164 - 174. 
[8] J. Meng, W. T Yin and Y. Y. Li, "Compressive Sensing Based High-Resolution Channel Estimation for OFDM System", IEEE Journal of Selected Topics in Signal Processing, vol. 6, no. 1, (2012), pp. 15 25.

[9] G. Taubock and F. Hlawatsch, "A compressed sensing technique for ofdm channel estimation in mobile environments: exploiting channel sparsity for reducing pilots", Proceedings of IEEE Int. Conf. Acoust. Speech, Signal Processing (ICASSP' 08), Las Vegas, NV, (2008), pp. 2885 - 2888.

[10] S. M. Kay, "Fundamentals of Statistical Signal Processing: Estimation Theory", Prentice-Hall, (1998).

[11] J. Oliver, R. Aravind and K. Prabhu, "Sparse channel estimation in OFDM systems by threshold-based pruning”, Electronics Letters, vol. 44, no. 13, (2008), pp. 830 - 832.

[12] H. B. Li, D. X Liu and J. Li, "Channel order and RMS delay spread estimation with application to AC power line communications”, Digital Signal Processing, no. 13, (2003), pp. 284 - 300.

[13] M. R. Raghavendra and K. Giridhar, "Improving channel estimation in OFDM system for sparse multipath channels", IEEE Signal Process letter. vol. 12, no. 1, (2005), pp. 52 - 55.

[14] B. Sheng, P. C. Zhu, X. H You, "A Low-Complexity Sparse Channel Estimation Method for OFDM Systems", IEICE transaction on communication. E93-B (8), (2010), pp. 2211 - 2214.

\begin{abstract}
Authors
Yuanhong Zhong, he received the B.S., M.S. and Ph.D. degrees in College of Communication Engineering, Chongqing University, in 2003, 2006, and 2011, respectively. His research includes digital and mobile communications, distributed video coding, Machine Vision and Wireless Multimedia Sensor Networks.

Yao Zhou, she received the B.S. in College of Communication Engineering, Chongqing University, in 2014. She is currently working toward the M.S. degree in communication engineering in Chongqing University of China.

Qilun Lei, she received the B.S. in College of Faculty of Mechanical\&Electronic Information, China University of Geosciences, in 2015. She is currently working toward the M.S. degree in communication engineering in Chongqing University of China.
\end{abstract}

